\title{
ALTERNATING BASIS EXCHANGES IN MATROIDS
}

\author{
JOSEPH P. S. KUNG
}

Abstract. We study some of the basis exchange properties for matroids implied by the following fact from linear algebra: an alternating multilinear $k$-form vanishes on any ordered set of $k$ linearly dependent vectors.

Let $X$ be a vector space of dimension $d$, and let

$$
\left[x_{1}, \ldots, x_{d}\right]=\operatorname{det}\left(x_{i j}\right) \text {, }
$$

where $\left(x_{i j}\right)$ are the coordinates of the vector $x_{i}$ in some coordinate system. Consider the alternating $p$-form $\mathrm{f}$ defined on a $p$-tuple of vectors $x_{1}, \ldots, x_{p}$ by:

$$
\begin{aligned}
& \mathbf{f}\left(x_{1}, \ldots, x_{p}\right) \\
& \quad=\sum_{\sigma} \operatorname{sgn} \sigma\left[x_{\sigma 1} \cdots x_{\sigma k} a_{k+1} \cdots a_{d}\right]\left[x_{\sigma k+1} \cdots x_{\sigma k+l} b_{l+1} \cdots b_{d}\right] \\
& \quad \cdots\left[x_{\sigma k+l+\cdots+m+1} \cdots x_{\sigma k+l+\cdots+m+n} c_{n+1} \cdots c_{d}\right],
\end{aligned}
$$

where (a) $p=k+l+\cdots+m+n$, (b) the vectors $a_{k+1}, \ldots, c_{d}$ are chosen constant vectors, and (c) the summation is over all shuffles $\sigma$ of $\{1, \ldots, p\}$ relative to the partition $\{\{1, \ldots, k\},\{k+1, \ldots, k+$ $l\}, \ldots,\{k+l+\cdots+m+1, \ldots, p\}\}$. Recall that if $S$ is a finite set, and $\pi=\left\{B_{1}, \ldots, B_{m}\right\}$ is a partition of $S$ into blocks $B_{i}$, then a shuffle $\sigma$ of $S$ relative to $\pi$ is a permutation of $S$ surch that there exists $i$ and $x \in B_{i}$ such that $x \notin B_{i}$. That is, a shuffle is a permutation that actually moves an element from one block to another.

Observe now that as $\mathbf{f}$ is alternating, $\mathbf{f}$ is zero on any $p$-tuple of linearly dependent vectors: in particular, if one of the summands on the right is nonzero, there must be another nonzero summand to cancel it. This fact, translated into the purely combinatorial language of matroid theory (for which we refer the naive reader to Crapo and Rota [1] and Whitney [5]), becomes

THEOREM 1. Let $B_{1}, \ldots, B_{m}$ be bases of a matroid $G(S)$ on the finite set $S$, with each basis partitioned into two blocks:

$$
B_{i}=X_{i} \cup Y_{1}, \quad 1 \leqslant i \leqslant m,
$$

Received by the editors September 20, 1977 and, in revised form, March 2, 1978.

AMS (MOS) subject classifications (1970). Primary 05B35; Secondary 15A03, $15 A 15$.

Key words and phrases. Matroid, combinatorial geometry, basis exchange, alternating multilinear form. 
such that the sets $Y_{i}$ are pairwise disjoint. Suppose that $Y=Y_{1} \cup \cdots \cup Y_{m}$ is dependent in $G(S)$. Then, there exists a shiuffle $\sigma$ with respect to the partition $\left\{Y_{1}, \ldots, Y_{m}\right\}$ such that, for all $i, X_{i} \cup \sigma Y_{i}$ is also a basis.

Our proof uses the "arrow" notation introduced in Greene and Magnanti [3] and Lawler [4]. We first construct the exchange graph, which is a way of encoding all the possible basis exchanges. The exchange graph is the directed graph (without loops) defined on the vertex set $Y$ as follows. Let $(a, b)$ be an ordered pair; as the sets $Y_{i}$ are pairwise disjoint, $b$ is in a unique basis $B_{i}$. The pair $(a, b)$ is an arc in the exchange graph if $\left(B_{i} \backslash b\right) \cup a$ is a basis. This situation is indicated by

$$
\underset{B_{i}}{a} b .
$$

Evidently, $a \rightarrow_{B_{i}} b$ whenever one of the following two equivalent conditions holds:

(i) $b$ is in the fundamental circuit of $a$ relative to $B_{i}$,

(ii) $a$ is contained in the bond $S \backslash \overline{\left(B_{i} \backslash b\right)}$.

Now, as $Y$ is dependent, $Y$ contains a circuit $C$. Since a bond is the complement of a closed set, the intersection of the circuit $C$ with any bond $D$ cannot be a single element set: that is,

$$
|C \cap D| \neq 1 \text {. }
$$

For $a \in C$ such that $a \in B_{i}$, consider the bond $D_{a}=S \backslash \overline{\left(B_{i} \backslash a\right)}$. As $a$ is assuredly contained in $C \cap D_{a}$, there must be, by our observation, another point $b \in C$, with $b \in B_{j}$, such that $b$ is in the bond $D_{a}$; that is to say,

$$
b \underset{B_{j}}{\rightarrow} a .
$$

Thus, from any point in $C$, we can always retreat to another point in $C$ through an arc of the exchange graph. As $C$ is finite, this implies that the exchange graph contains a directed cycle.

Choose a directed cycle of minimum length:

$$
\underset{B_{i}}{\rightarrow} \underset{B_{j}}{\rightarrow} c \cdots d \underset{B_{k}}{\rightarrow} a .
$$

The permutation $\sigma$ on $Y$, defined by sending $a$ to $b, b$ to $c, \ldots$ while keeping the points not in the cycle fixed, is in fact a shuffle, since consecutive arcs in the cycle cannot be labelled by the same basis. Moreover, for all $i, X_{i} \cup \sigma Y_{i}$ is a basis; this is proved in [3], but the proof is worth repeating.

Let $x_{p} \rightarrow_{B_{i}} y_{p}, 1 \leqslant p \leqslant n$, be all the arcs in the cycle labelled $B_{i}$, arranged in their order of appearance from left to right in (*). We claim that for all $p$,

$$
B_{i, p}=\left(B_{i} \backslash\left\{y_{1}, \ldots, y_{p}\right\}\right) \cup\left\{x_{1}, \ldots, x_{p}\right\}
$$

is a basis. For $p=1$, this follows from the definition. Suppose that this holds up to $q-1$. Note that the fundamental circuit of $x_{q}$ relative to $B_{i}$ is contained in $\left(B_{i} \backslash\left\{y_{1}, \ldots, y_{q-1}\right\}\right) \cup x_{q}$; since, if it were not, there would be an $r, 1 \leqslant r \leqslant q-1$, such that $x_{q} \rightarrow_{B_{i}} y_{r}$, contradicting the fact that the cycle is of minimum size. Moreover, the fundamental circuits of $x_{q}$ relative to $B_{i}$ 
and $B_{i, q-1}$ are the same. Hence, we can replace $y_{q}$ by $x_{q}$ in $B_{i, q-1}$ to obtain the basis $B_{i, q}$. Our claim now follows by induction. Setting $p=n$, we conclude that $B_{i, n}=X_{i} \cup \sigma Y_{i}$ is a basis. The proof of the theorem is now complete.

A special case of the theorem is obtained if we postulate that $|Y|>\operatorname{rank} G$ +1 ; this was first proved in [2] and may be regarded as a generalisation of the basis exchange axiom.

The roles played by circuits and bonds are symmetrical in the proof. Thus, duality provides us with another metamorphosis:

COROLlaRY 2. Under the same initial hypotheses, suppose that $X=X_{1}$ $\cup \cdots \cup X_{m}$ is a nonspanning set. Then, there exists a shuffle $\sigma$ of $Y$ with respect to the partition $\left\{Y_{1}, \ldots, Y_{m}\right\}$ such that, for all $i, X_{i} \cup \sigma Y_{i}$ is also a basis.

A further analysis of the proof yields a third metamorphosis:

THEOREM 3. Let $B_{1}, \ldots, B_{m}$ be bases of the matroids $G_{1}(S), \ldots, G_{m}(S)$ on the finite set $S$, with each basis partitioned into two blocks:

$$
B_{i}=X_{i} \cup Y_{i}, \quad 1 \leqslant i \leqslant m,
$$

such that the sets $Y_{i}$ are pairwise disjoint. Suppose that $Y=Y_{i} \cup \cdots \cup Y_{m}$ contains a subset $Y^{\prime}$ such that $Y^{\prime}$ is a union of circuits in each of the matroids $G_{i}(S), 1 \leqslant i \leqslant m$. Then, there exists a shuffle $\sigma$ with respect to the partition $\left\{Y_{1}, \ldots, Y_{m}\right\}$ such that, for all $i, X_{i} \cup \sigma Y_{i}$ is a basis in the matroid $G_{i}(S)$.

The proof is a straightforward modification of the proof of the first theorem. The dual version of Theorem 3 is left as an exercise.

The final version of the alternating basis exchange property has an interesting interpretation in terms of matrices. Let $M$ be a matrix with rows indexed by $T$ and columns indexed by $S$. For a subset $U$ of $T$, let $M \mid U$ be the matrix obtained from $M$ by deleting all the rows not in $U$. We denote by $G \mid U$ the matroid defined on $S$ (in the usual fashion) by the matrix $M \mid U$.

THEOREM 4. Let $B_{1}$ and $B_{2}$ be bases of the matroids $G \mid U$ and $G \mid V$. Suppose that $A_{1} \subseteq B_{1}, A_{2} \subseteq B_{2}$ are subsets such that $A_{1} \cap A_{2}=\varnothing$ and there exists no shuffle $\sigma$ of $A_{1} \cup A_{2}$ relative to $\left\{A_{1}, A_{2}\right\}$ such that $B_{1} \backslash A_{1} \cup \sigma A_{1}$ and $B_{2} \backslash A_{2}$ $\cup \sigma A_{2}$ are both bases. Then, $A_{1} \cup A_{2}$ is an independent set in $G \mid U \cup V$.

Proof. We note that $G \mid U$ and $G \mid V$ are projections (or, in matroidal terminology, strong map images) of $G \mid U \cup V$. Under a projection, any circuit is projected onto a union of circuits. Thus, if there exists no shuffle of the form specified, $A_{1} \cup A_{2}$ cannot contain a circuit in $G \mid U \cup V$.

ACKNowledgement. I thank Gian-Carlo Rota for many helpful discussions.

\section{REFERENCES}

1. H. H. Crapo and G.-C. Rota, On the foundations of combinatorial theory: Combinatorial geometries, M. I. T. Press, Cambridge, Mass., 1970.

2. C. Greene, Another exchange property for bases, Proc. Amer. Math. Soc. 46 (1974), 155-156. 
3. C. Greene and T. L. Magnanti, Some abstract pivot algorithms, SIAM J. Appl. Math. 29 (1975), 530-539.

4. E. L. Lawler, Combinatorial optimization: Networks and matroids, Holt, Rinehart and Winston, New York, 1976.

5. H. Whitney, The abstract properties of linear dependence, Amer. J. Math. 57 (1935), 507-533.

Department of Mathematics, Massachusetts institute of Technology, Cambridge, MASSACHUSETTS 02139 